\title{
Selective Inhibition of $\beta$-Catenin/Co-Activator Cyclic AMP Response Element-Binding Protein-Dependent Signaling Prevents the Emergence of Hapten-Induced Atopic Dermatitis-Like Dermatitis
}

\author{
Haruna Matsuda-Hirose ${ }^{1}$, Tomoko Yamate ${ }^{1}$, Mizuki Goto ${ }^{1}$, Akira Katoh ${ }^{2}$, Hiroyuki Kouji ${ }^{2}$, \\ Yuya Yamamoto ${ }^{1}$, Takashi Sakai ${ }^{1}$, Naoto Uemura ${ }^{3}$, Takashi Kobayashi ${ }^{4}$, Yutaka Hatano ${ }^{1}$ \\ ${ }^{1}$ Department of Dermatology and ${ }^{2}$ Translational Chemical Biology, Departments of ${ }^{3}$ Clinical Pharmacology and Therapeutics and \\ ${ }^{4}$ Infectious Disease Control, Faculty of Medicine, Oita University, Oita, Japan
}

Background: The canonical Wnt/ $\beta$-catenin signaling pathway is a fundamental regulatory system involved in various biological events. ICG-001 selectively blocks the interaction of $\beta$-catenin with its transcriptional co-activator cyclic AMP response element-binding protein (CBP). Recent studies have provided convincing evidence of the inhibitory effects of ICG-001 on Wnt-driven disease models, such as organ fibrosis, cancer, acute lymphoblastic leukemia, and asthma. However, the effects of ICG-001 in atopic dermatitis (AD) have not been investigated. Objective: To investigate whether $\beta$-catenin/CBP-dependent signaling was contributed in the pathogenesis of AD and ICG-001 could be a therapeutic agent for AD. Methods: We examined the effects of ICG-001 in an AD-like murine model generated by repeated topical application of the hapten, oxazolone (Ox). ICG-001 or vehicle alone was injected intraperitoneally every day during the development of AD-like dermatitis arising from once-daily Ox treatment. Results: Ox-induced AD-like dermatitis

Received May 16, 2019, Revised August 15, 2019, Accepted for publication September 6, 2019

Corresponding author: Yutaka Hatano, Department of Dermatology, Faculty of Medicine, Oita University, 1-1 Idaigaoka, Hasama-machi, Yufu, Oita 879-5593, Japan. Tel: 81-97-586-5882, Fax: 81-97-586-5889, E-mail: hatano@oita-u.ac.jp

ORCID: https://orcid.org/0000-0002-1349-755X

This is an Open Access article distributed under the terms of the Creative Commons Attribution Non-Commercial License (http://creativecommons. org/licenses/by-nc/4.0) which permits unrestricted non-commercial use, distribution, and reproduction in any medium, provided the original work is properly cited.

Copyright (c) The Korean Dermatological Association and The Korean Society for Investigative Dermatology characterized by increases in transepidermal water loss, epidermal thickness, dermal thickness accompanied by increased myofibroblast and mast cell counts, and serum levels of thymic stromal lymphopoietin and thymus and activation-regulated chemokine, and decreases in stratum corneum hydration, were virtually normalized by the treatment with ICG-001. Elevated serum levels of periostin tended to be downregulated, without statistical significance. Conclusion: These results suggest that $\beta$-catenin/CBP-dependent signaling might be involved in the pathogenesis of $A D$ and could be a therapeutic target. (Ann Dermatol 31(6) 631 639, 2019)

-Keywords-

Atopic dermatitis, Mouse, Therapy

\section{INTRODUCTION}

Atopic dermatitis (AD) is a chronic inflammatory skin disease and the pathogenesis of AD is composed of elements of barrier dysfunction and alterations in cell-mediated immune responses ${ }^{1,2}$. Permeability barrier dysfunction is observed in lesional as well as non-lesional skin. The imbalance of $\mathrm{T}$ helper (Th) 2 to Th1 cytokines observed in AD can create alterations in cell-mediated immune responses. Skin fibrotic remodeling is also one of the major pathological features of $A D$, especially in its chronic phase $^{3}$. Several studies have indicated that extracellular matrix components induce processes that result in the development of dermal fibrosis, and activate or protract the immune response. For example, periostin secreted from fi- 
broblasts, an extracellular matrix protein induced by Th2 cytokines, promotes chronic allergic inflammation ${ }^{4}$. All of these appear to play roles in the development of AD. Although the pathophysiology of AD is characterized by structural abnormalities in the epidermis and immune dysregulation, the complex pathophysiology of $A D$ is still not completely understood. In fact, incomplete efficacy of dupilumab ${ }^{5}$, clearly demonstrate complexity of the pathogenesis of $A D$ in which a variety of factors other than Th2 cytokines should be involved.

The canonical $\mathrm{Wnt} / \beta$-catenin signaling pathway is a fundamental mechanism accounting for various biological activities, such as cell proliferation, differentiation, and development. Levels of $\beta$-catenin are regulated by the $\mathrm{Wnt} /$ $\beta$-catenin pathway. In the inactive state, the $\beta$-catenin protein is degraded by a destruction complex composed of AXIN, glycogen synthase kinase $3 \beta($ GSK3 $\beta$ ) and adenomatous polyposis coli (APC). On the activation of the Wnt $\beta$ catenin pathway, the AXIN GSK3 $\beta$-APC complex is disrupted and GSK3 $\beta$ is inactivated, resulting in the protein stabilization and nuclear translocation of $\beta$ -catenin. In the nucleus, $\beta$-catenin stimulates the transcription of target genes in cooperation with T-cell-factor/lymphoid enhancer-binding factor. To generate a transcriptionally active complex, $\beta$-catenin recruits the transcriptional coactivators, cyclic AMP response element-binding protein-binding protein $(\mathrm{CBP})$ or its closely related homolog, p300, as well as other components of the basal transcription machinery, leading to the expression of a host of downstream target genes ${ }^{6}$. Aberrant $\mathrm{Wnt} \beta$-catenin signaling has been suggested to be involved in a variety of human pathologies, including cancers and metabolic, inflammatory, and fibrotic diseases ${ }^{7}$. ICG-001 selectively blocks the interaction of $\beta$-catenin with CBP. Recent studies have provided convincing evidence of the inhibitory effects of ICG-001 on disease models driven by Wnt signaling such as cancer ${ }^{8}$, organ fibrosis $^{9}$, and acute lymphoblastic leukemia ${ }^{10}$, and, in particular, the improvement of asthma ${ }^{11}$. However, the effects of ICG-001 on AD have not been examined. Further, the mechanisms of ICG-001 in allergic disease are not yet fully understood. In this study, we investigated the efficacy of ICG-001 in a mouse model of AD-like dermatitis.

\section{MATERIALS AND METHODS}

\section{Animals}

Female hairless (Hr-/Kud) mice (8 weeks old, 20 23 g body weight) were purchased from Kyudo Company. (Fukuoka, Japan). The animals were group-housed, four mice per cage, under conventional conditions and had ad libitum access to a commercial diet and water. All experiments with mice were approved by the Ethics of Animal Experimentation Committee at Oita University (approval no. 1725002).

\section{Development of oxazolone-induced AD-like dermatitis}

Development of a hapten (oxazolone, Ox)-induced murine model with lots of features of AD (Ox-AD) was described in our previous studies ${ }^{12,13}$. Animals were sensitized by two consecutive days of topical treatment with 50 $\mu \mathrm{l}$ of $5 \%$ Ox in acetone. After one week, mice were treated topically on both flanks with a total of $100 \mu \mathrm{l}$ of $1 \%$ $\mathrm{Ox}$ in ethanol once every other day for an additional 3 weeks (total of 10 challenges).

\section{ICG-001 administration and tissue preparation}

ICG-001 was manufactured and provided by Prof. Kouji (Oita University, Japan). Mice were injected intraperitoneally with ICG-001 (4 mg/kg body weight) diluted in vehicle, i.e., dimethyl sulfoxide (DMSO), PEG300, Tween80 , and $\mathrm{dH}_{2} \mathrm{O}$, or vehicle alone (vehicle control) once daily in an injection volume of $50 \mu \mathrm{I}$. On the last day of the experiment (day 29), the animals were sacrificed by cervical dislocation after transepidermal water loss (TEWL) and stratum corneum (SC) hydration were measured, and serum and skin samples were collected for the measurements and assessments described below.

\section{Physiological assessments}

TEWL and SC hydration on flanks were respectively measured using a Tewameter (TM300; Courage \& Khazaka Electronic, Köln, Germany) and a Corneometer (CM825; Courage \& Khazaka Electronic).

\section{Histological analysis}

Collected skin samples were fixed in $10 \%$ formalin and embedded in paraffin blocks. Tissue sections (5- $\mu \mathrm{m}$ thickness) were stained with hematoxylin-eosin for histological observation or Giemsa-stain for identification of mast cells, and then examined under light microscopy (Olympus, Tokyo, Japan). Epidermal and dermal thickness at six different locations on each image were measured as the distance from the basement membrane to the outer edge of the epidermis and from the epidermal-dermal junction to the dermal-subcutaneous fat junction, respectively, using a fluorescence microscope (BZ-X700; Keyence Corp., Osaka, Japan) at a magnification of $400 \times$ by an investigator blinded to the identity of the samples.

\section{Immunohistochemistry}

To detect myofibroblasts, skin sections were stained with 
a monoclonal antibody against $\alpha$-smooth muscle actin (clone 1A4; Sigma-Aldrich, Taufkirchen, Germany). To examine whether $\mathrm{CBP} / \beta$-catenin is involved in dermal fibrosis $^{14}$, skin sections were stained with a monoclonal antibody against S100A4 (ab197896; Abcam, Cambridge, UK). Paraffin sections (5- $\mu \mathrm{m}$ thickness) were deparaffinized and incubated with a monoclonal antibody against $\alpha$ smooth muscle actin and S100A4 overnight at $4^{\circ} \mathrm{C}$. Detection was performed using an horseradish peroxidase (HRP) -conjugated secondary antibody (Dako envision + dual link system-HRP (diaminobenzidine+, DAB +); Dako, Carpinteria, CA, USA) or (Hystfine simple stain MAX-PO(R); Nichirei, Tokyo, Japan) followed by chromogenic detection using DAB (Dojin Chem., Kumamoto, Japan) as the substrate.

\section{Counts of myofibroblasts and mast cells in the murine dermis}

For quantification of myofibroblasts positive for $\alpha$-smooth muscle actin, spindle-shaped cells in the dermis and mast cells in the Giemsa-stained dermis were counted in one or two randomly chosen high-power fields (magnification $\times 400$ ) in each mouse by two experienced researchers in a blinded manner.

\section{Cell culture}

Culture of normal human keratinocytes (NHKs) (cat. no. 102-05; Cell Applications, Inc., San Diego, CA, USA) was performed as previously reported ${ }^{15,16}$. When NHKs reached $70 \%$ to $90 \%$ confluence, expression of thymic stromal lymphopoietin (TSLP) was induced by stimulation with poly I:C (10 $\mu \mathrm{g} / \mathrm{ml})$, tumor necrosis factor (TNF)- $\alpha$ (20 $\mathrm{ng} / \mathrm{ml}$; R\&D Systems, Minneapolis, MN, USA), and interleukin (IL)-4 (100 ng/ml; R\&D Systems) with or without ICG-001 (5 $\mu \mathrm{M})$. Hydrocortisone-free media were used during the stimulation protocol. The supernatants for enzyme-linked immunosorbent assay (ELISA) and NHKs for real-time polymerase chain reaction (PCR) were harvested 24 hours and 6 hours, respectively, after the stimulation. Normal human dermal fibroblasts (NHDFs) (CSC-2F0; Cell Systems, Kirkland, WA, USA) were cultured in Dulbecco's modified eagle medium supplemented with $10 \%$ fetal bovine serum, $100 \mathrm{U} / \mathrm{ml}$ of penicillin, and $100 \mu \mathrm{g} / \mathrm{ml}$ of streptomycin at $37^{\circ} \mathrm{C}$ in a humidified atmosphere of $5 \%$ $\mathrm{CO}_{2}$ and $95 \%$ air. The cells were cultured at an initial density of $5 \times 10^{4}$ cells $/ \mathrm{ml}$ overnight. The medium was then replaced with serum-free medium and stimulated with human recombinant IL-4 (100 ng/ml; R\&D Systems), IL-13 (100 ng/ml; R\&D Systems) and with or without ICG-001 (5 $\mu \mathrm{M})$ diluted in DMSO. The final concentration of DMSO was $0.1 \%$. An equivalent concentration of DMSO $(0.1 \%)$ was used as the vehicle medium in all experimental groups including the negative control. The supernatants for ELISA and NHDFs for real-time PCR were harvested 24 hours after stimulation.

\section{mRNA isolation, reverse transcription, and real-time PCR}

RNA isolation from skin samples minced in liquid nitrogen was performed using TRIzol reagent (Invitrogen, Karlsruhe, Germany) and the PureLink ${ }^{\circledR}$ RNA Mini Kit (Ambion, Thermo Fisher Scientific, Waltham, MA, USA). mRNA isolation from cultured NHKs and NHDFs was performed using the High Pure RNA Isolation Kit. mRNA was reverse transcribed to cDNA with Transcriptor First Strand cDNA Synthesis Kit (Roche Diagnostics, Basel, Switzerland). cDNA was analyzed by real-time PCR (The LightCycler ${ }^{\circledR} 96$ System; Roche Diagnostics) using KAPA SYBR ${ }^{\circledR}$ FAST quantitative PCR master mix. Quantifications were normalized to the housekeeping gene, glyceraldehyde 3-phosphate dehydrogenase (GAPDH). Relative expression is given as the ratio between target gene expression and GAPDH expression. The primers used for real-time PCR are shown in Supplementary Table 1.

\section{Measurements of levels of TSLP, thymus and activation- regulated chemokine, periostin and immunoglobulin $E$}

Levels of TSLP, TARC, periostin and immunoglobulin $E$ (IgE) in serum were examined by ELISA using a mouse TSLP Quantikine ELISA Kit (R\&D Systems), a mouse CCL17/thymus and activation-regulated chemokine (TARC) Quantikine ELISA Kit (R\&D Systems), a mouse periostin/OSF-2 Quantikine ELISA Kit (R\&D Systems), and a mouse IgE quantitation kit (Yamasa Co., Ltd., Chiba, Japan) according to each manufacturer's instructions. Levels of TSLP and periostin in supernatant of cultured human keratinocytes or fibroblasts were determined by ELISA with human TSLP Quantikine ELISA Kit (R\&D Systems) and human Periostin/OSF-2 DuoSet ELISA (R\&D Systems) according to each manufacturer's instructions.

\section{Statistical analysis}

All experiments were analyzed using ANOVA with Tukey's multiple-comparison tests. All results are presented as mean \pm standard error of the mean. Values of $p<0.05$ were considered statistically significant.

\section{RESULTS}

ICG-001 attenuated AD-like skin dermatitis and epidermal permeability barrier function in Ox-AD mice

We examined the effect of ICG-001 in an AD-like model induced by repeated Ox treatments. ICG-001 or vehicle 
alone were injected intraperitoneally once daily (each group $n=8$ ). Forty-eight hours after the tenth Ox treatment to the sensitized mice, their skin appearance exhibited AD-like lesions such as erythema, edema, lichenification, and scale compared with the normal appearance of the non-treated control group (normal group $n=4$ ). ICG-001 attenuated Ox-AD skin lesions compared with vehicle group (Ox+vehicle; Fig. 1).

\section{ICG-001 reduced epidermal hyperplasia, dermal fibrosis, and inflammatory cell infiltration in AD-like skin}

Epidermal hyperplasia and skin fibrotic remodeling are major histopathological features in human AD. The application of ICG-001 suppressed epidermal hyperplasia and prevented the Ox-induced dermal fibrosis as assessed by the measurement of epidermal and dermal thickness and myofibroblast counts (Fig. 2A D). Accordingly, S100A4 expression, which is upregulated by $\mathrm{CBP} / \beta$-catenin ${ }^{14}$, was increased in Ox-induced dermal fibrosis, and this induction was reduced by the co-administration of ICG-001 (Supplementary Fig. 1).

AD-like skin showed marked dermal inflammatory cell infiltration. In particular, the numbers of mast cells in Giemsa stains were increased (Fig. 2E). ICG-001 treatment significantly suppressed the Ox-induced infiltration of mast cells.

\section{ICG-001 attenuated epidermal permeability barrier func- tion in Ox-AD mice}

Basal TEWL was significantly increased and SC hydration was significantly decreased in Ox-AD skin lesions. The ICG-001 group (Ox+ICG-001) decreased basal TEWL (Fig. 3A) and increased SC hydration (Fig. 3B) in Ox-AD skin lesions compared with the vehicle group $(\mathrm{Ox}+\mathrm{ve}-$ hicle; $p<0.01$ ).

\section{Effects of ICG-001 on serum levels of TSLP, TARC, IgE, and periostin in Ox-AD mice}

Levels of IgE and TSLP in serum were remarkably elevated in Ox-AD mice compared with control mice. Levels of TARC and periostin in serum also tended to be elevated in Ox-AD mice compared with control mice. ICG-001 treatment of AD mice suppressed serum levels of TSLP and TARC but not IgE. ICG-001 also tended to suppress serum levels of periostin, without statistical significance (Fig. 4). Moreover, mRNA expression of TSLP and periostin in lesional skin was not affected by ICG-001, as shown by real-time PCR in Supplementary Fig. 2.

\section{ICG-001 does not affect the expression of TSLP in cultured human keratinocytes}

TSLP expression induced by TNF- $\alpha$, IL-4, and poly:IC in NHKs was not affected by ICG-001 (Supplementary Fig. 3A, $B)$. These results indicate that ICG-001 does not have a direct effect on the expression and induction of TSLP in NHKs.

\section{ICG-001 inhibits IL-4-, IL-13-induced periostin pro- duction by cultured human dermal fibroblasts}

Next, we examined whether ICG-001 could inhibit IL-4, IL-13-induced periostin expression in NHDFs. Similar to our in vivo results, periostin mRNA expression was not affected by ICG-001, as shown by real-time PCR (Supplementary Fig. 3C). Furthermore, consistent with the observed trend in vivo, the level of periostin in the culture supernatant was significantly decreased, as shown by

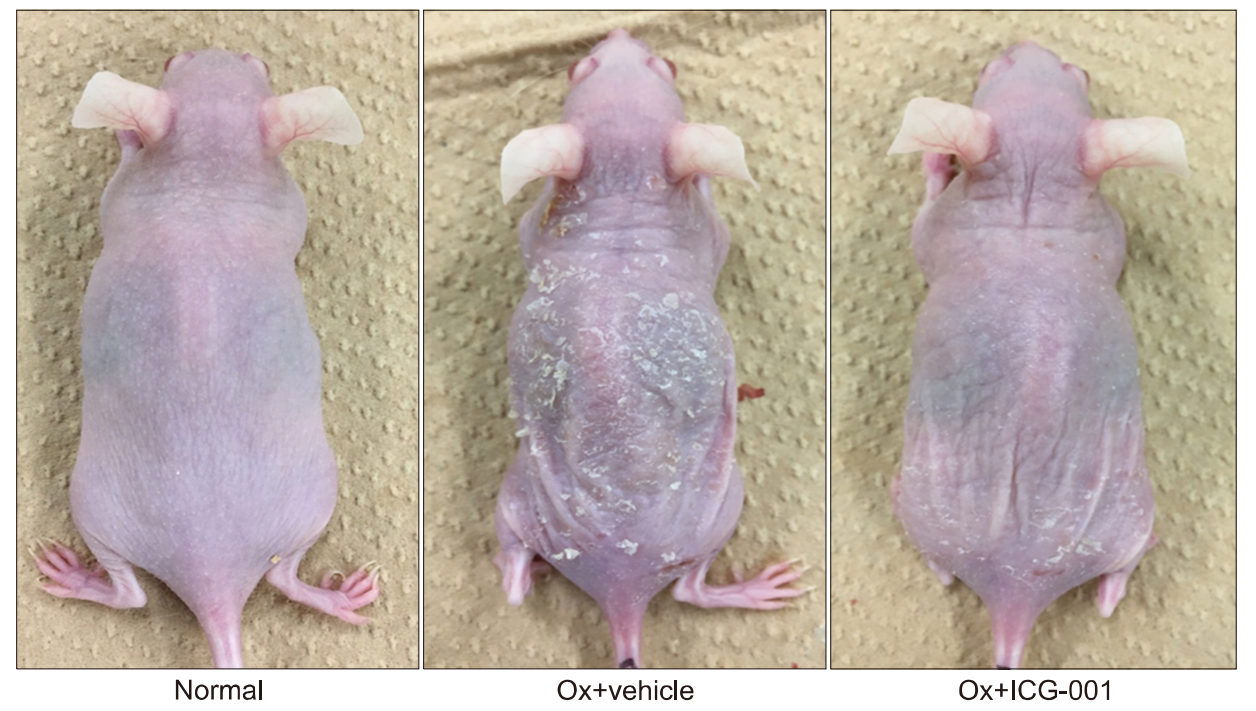

Fig. 1. Effect of ICG-001 in an atopic dermatitis (AD)-like model induced by repeated oxazolone (Ox) treatments. As Ox treatments proceeded, the gross appearances of $\mathrm{AD}$ lesions showing remarkable erythema, lichenification, and pronounced scales compared with the non-treated control group (normal). ICG-001 treatment (Ox+ICG-001) remarkably attenuated AD-like skin lesions compared with vehicle only mice $(\mathrm{Ox}+$ vehicle). 


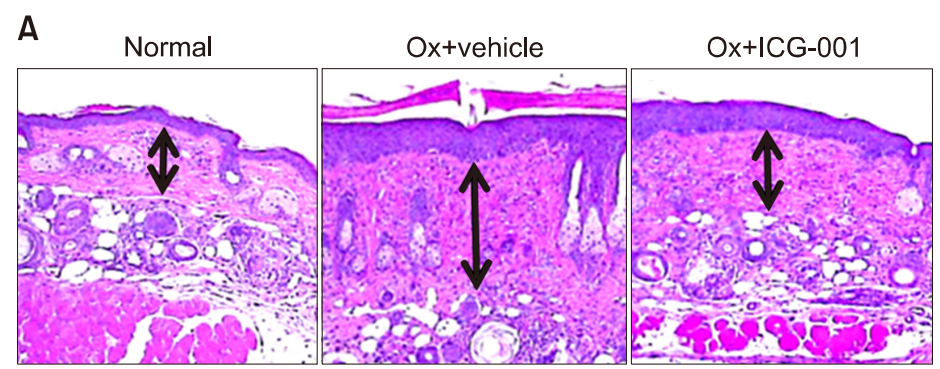

B

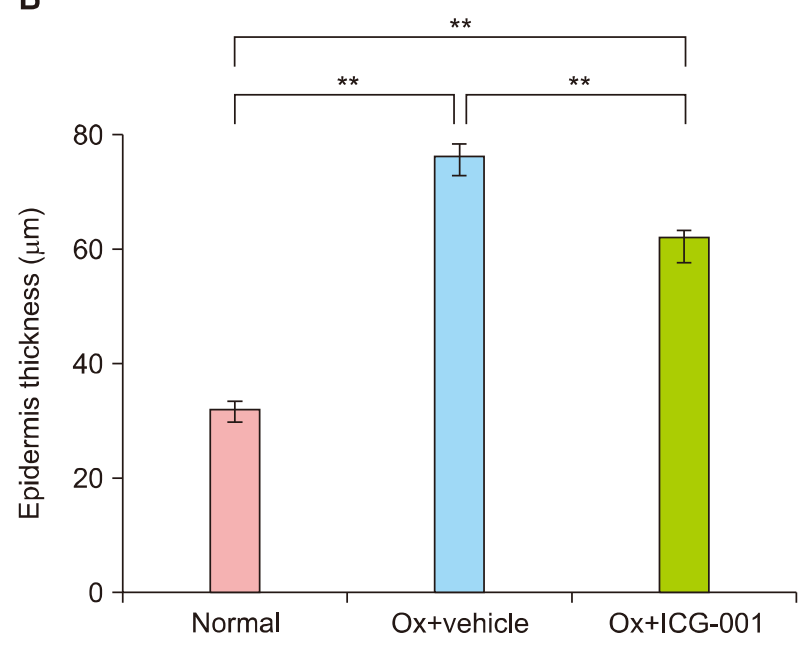

D

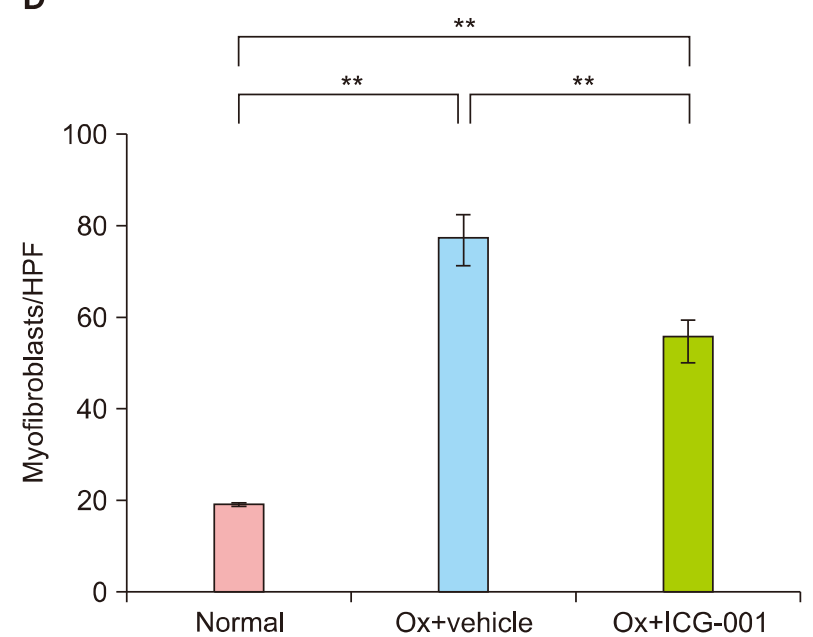

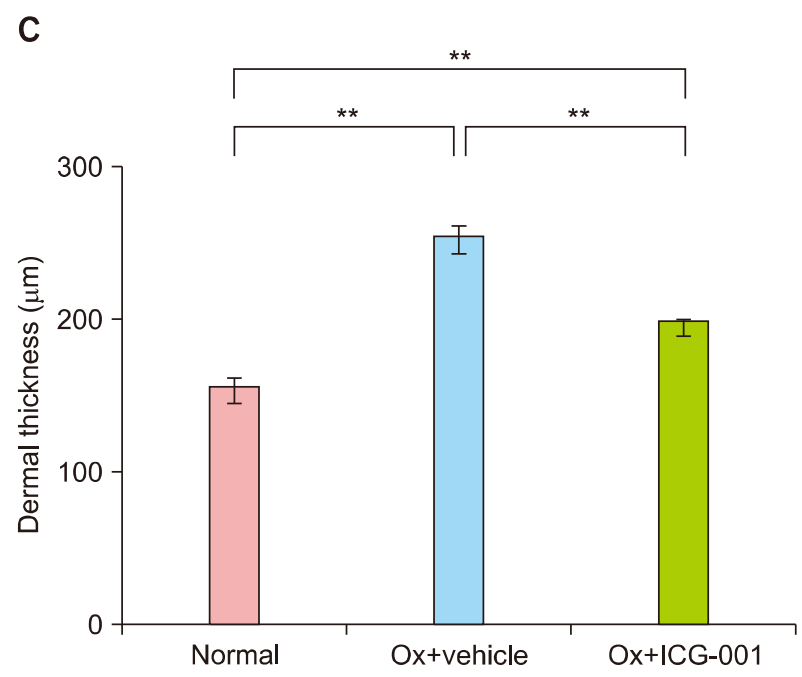

E

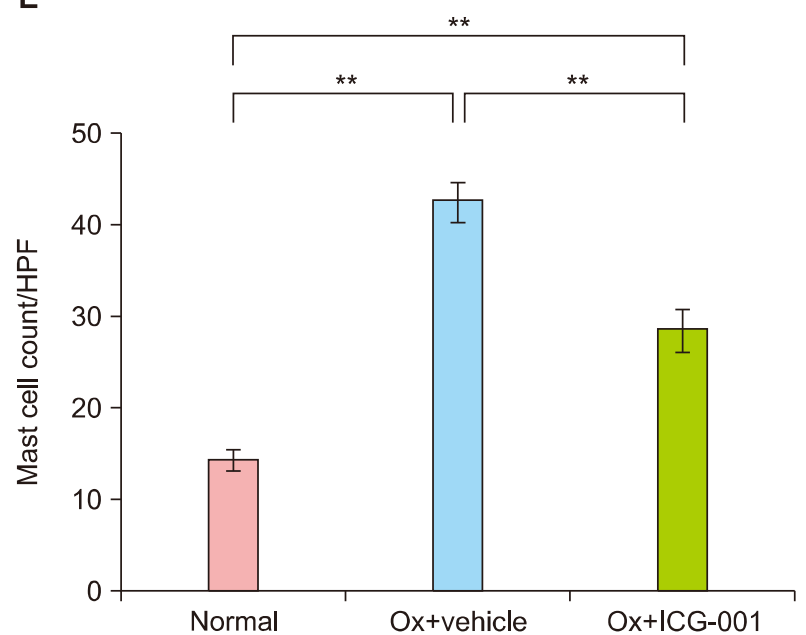

Fig. 2. Effects of ICG-001 on epidermal hyperplasia, dermal fibrosis, and inflammatory cell infiltration in atopic dermatitis (AD)-like skin. (A) H\&E stained sections are shown at 100x magnification. (B, C) Skin thickening as determined by H\&E staining. ICG-001 suppressed epidermal hyperplasia and dermal fibrosis induced by repeated oxazolone (Ox) treatment. (D, E) $\alpha$-Smooth muscle actin (SMA) positive myofibroblasts by immunohistochemical staining and mast cells by Giemsa-staining per high power field. $* * p<0.01$. ICG-001 significantly suppressed myofibroblast proliferation and mast cell infiltration in the dermis. Control group (normal) ( $\mathrm{n}=4$ ), Vehicle only mice $\left(\mathrm{Ox}+\right.$ vehicle) $(\mathrm{n}=8)$, ICG-001 treatment $(\mathrm{Ox}+\mathrm{ICG}-001)(\mathrm{n}=8) .{ }^{*} 0.05>p>0.01,{ }^{* *} p<0.01$.

ELISA (Supplementary Fig. 3D), accompanied by a decrease in fibroblast counts (Supplementary Fig. 4).

\section{DISCUSSION}

The effects of ICG-001 on Ox-induced AD, namely decreases in TEWL, mast cell numbers in the dermis, epi- 
A

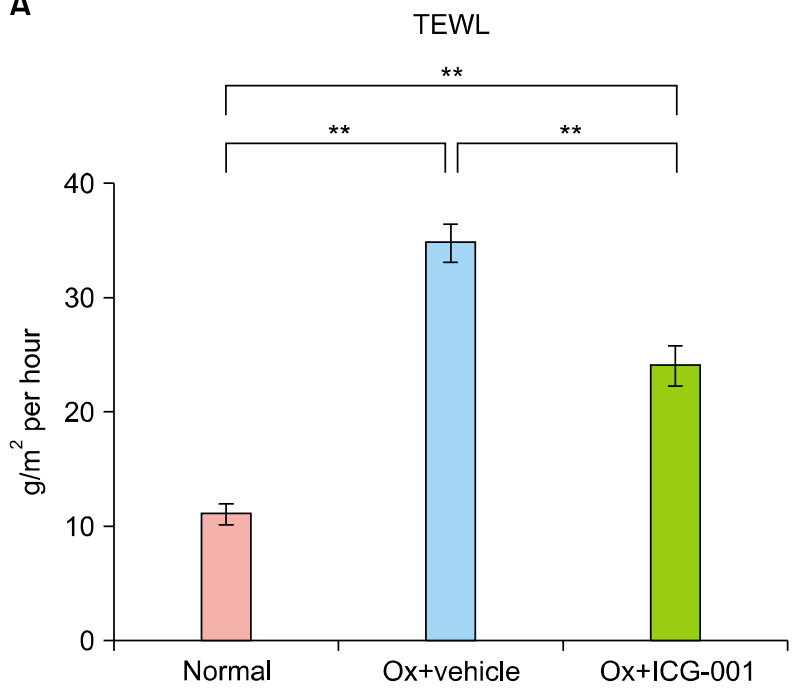

B

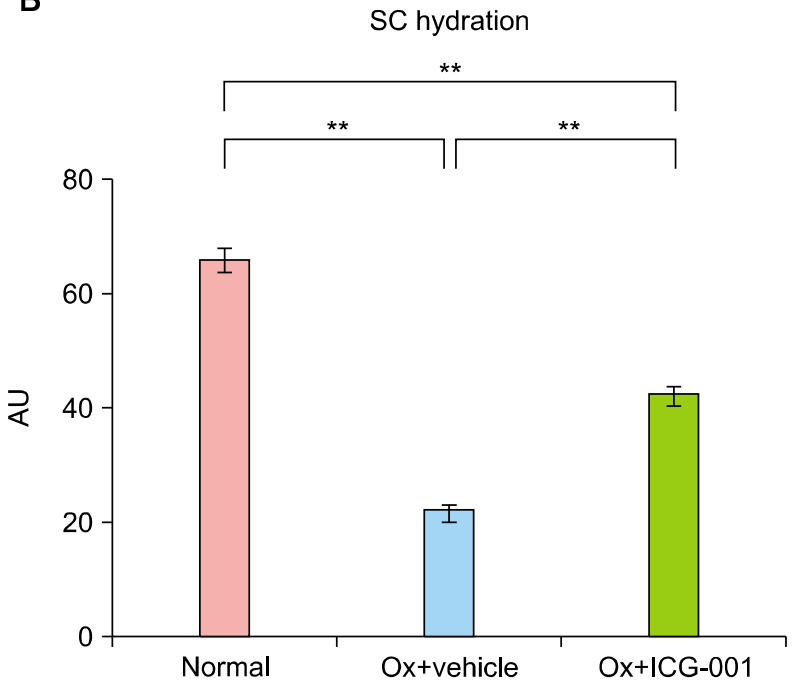

Fig. 3. Effect of ICG-001 on epidermal permeability barrier function in atopic dermatitis (AD)-like skin. ICG-001 treated mice (oxazolone $[\mathrm{Ox}]+\mathrm{ICG}-001$ ) showed lower basal transepidermal water loss (TEWL) (A) and higher stratum corneum (SC) hydration (B) compared with vehicle only mice $(\mathrm{Ox}+$ vehicle). Control group (normal) $(\mathrm{n}=4)$, Vehicle only mice $(\mathrm{Ox}+$ vehicle) $(\mathrm{n}=8)$, ICG-001 treatment $(\mathrm{Ox}+\mathrm{ICG}-001)(\mathrm{n}=8) .{ }^{*} 0.05>p>0.01,{ }^{* *} p<0.01$.

dermal and dermal thicknesses, serum levels of TARC and TSLP, and the increase in SC hydration, suggest that $\beta$ catenin/CBP-dependent signaling could be involved in permeability barrier dysfunction, allergic inflammation, and skin fibrotic remodeling in the pathogenesis of AD. Levels of S100A4 expression in skin were increased in Ox-induced $A D$ and those were downregulated by the administration of ICG-001. These results suggest not only that $\beta$-catenin/CBP-dependent signaling is involved in the pathogenesis of Ox-induced AD but also that ICG-001 successfully inhibited the signaling in the present study. Further studies to check the involvement of $\beta$-catenin/ CBP-dependent signaling in the pathogenesis of human $A D$ should be also conducted in future.

ICG-001 is shown to have efficacy in various models driven by Wnt signaling, in particular organ fibrosis such as bleomycin-induced lung fibrosis or airway remodeling in asthma ${ }^{7}$. Therefore, the decrease in dermal thickness accompanying the decrease in dermal myofibroblast numbers might be a direct effect of ICG-001. Accordingly, ICG-001 inhibited increases in cultured human fibroblasts induced by IL-4 and IL-13 in the present study. However, mRNA expression of periostin in skin lesions and cultured human fibroblasts was not affected by ICG-001 treatment. Therefore, down-regulation of periostin in the supernatant of cultured fibroblasts and the tendency for decreased serum levels of periostin following ICG-001 treatment might be due to inhibition of fibroblast proliferation but not down-regulation of mRNA expression of periostin in fibroblasts. Furthermore, it was reported that upregulation of periostin mRNA expression by transforming growth factor (TGF) - $\beta$ in cultured murine $3 \mathrm{~T} 3$ fibroblasts was inhibited by ICG- $001{ }^{17}$, suggesting that fibroblasts treated with IL-4 and IL-13 might respond differently to ICG-1 compared to fibroblasts treated with TGF- $\beta$.

Accompanying the reduction of AD-like lesions, serum levels of TSLP were down-regulated by ICG-001 treatment. On the other hand, mRNA expression of TSLP in cultured human keratinocytes was not affected by ICG-001, suggesting that the decrease in serum levels of TSLP in ICG001-treated mice was not a direct effect of ICG-001 on keratinocytes. Furthermore, mRNA expression of TSLP was not elevated in Ox-induced AD-like lesions, which were not affected by ICG-001 in the present study. It has been demonstrated that TSLP mRNA expression is induced transiently after stimulation ${ }^{18}$. Therefore, we speculate that skin samples harvested 48 hours after Ox treatment might not be suitable for evaluating TSLP mRNA expression.

In spite of the direct or secondary modulatory effects of ICG-001 on serum levels of periostin and TSLP, serum levels of IgE were not significantly affected by ICG-001. These findings contradict previous reports that periostin produced by fibroblasts acts on keratinocytes, inducing production of TSLP for Th2 skewing and, as a result, the cycle between Th2 and periostin maintains persistent inflammation in AD. A possible explanation underlying this discrepancy is presented below. Wnt signaling results in upregulation of SATB1 to recruit the $\beta$-catenin-p300 complex, which drives expression of GATA-3 in Th2 cells, 
A

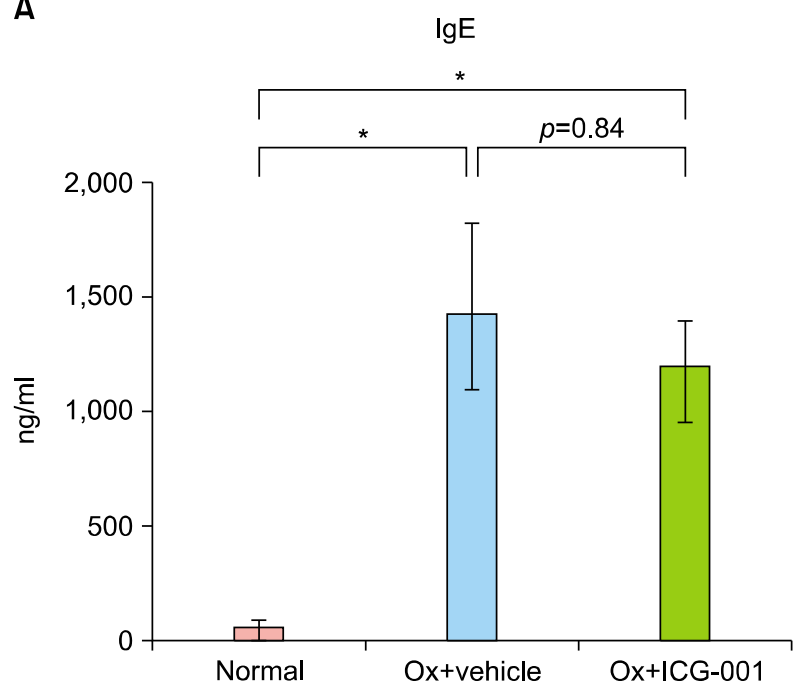

C

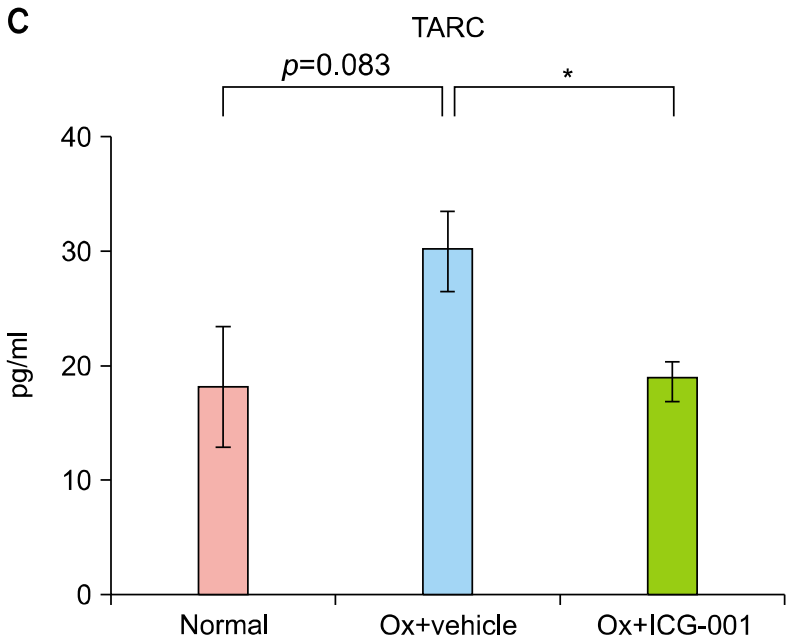

B

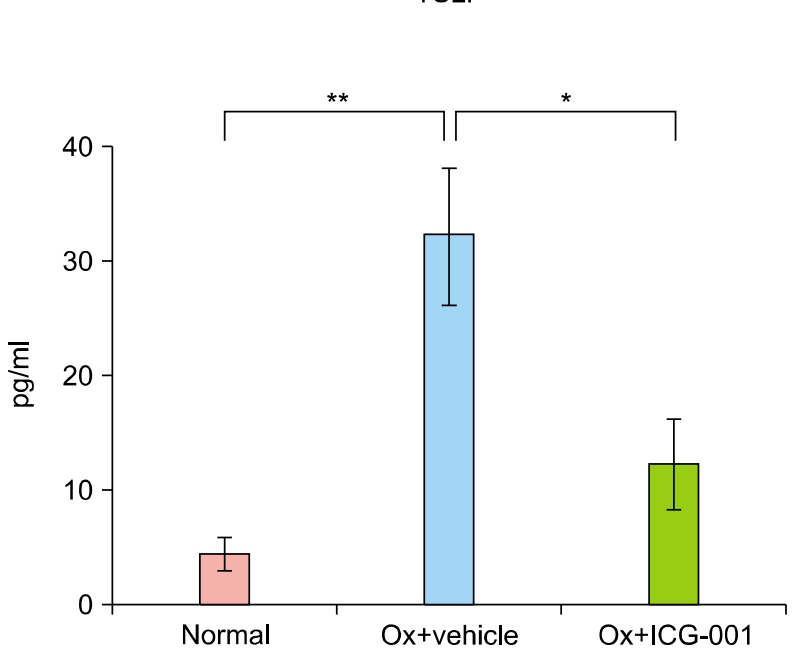

D

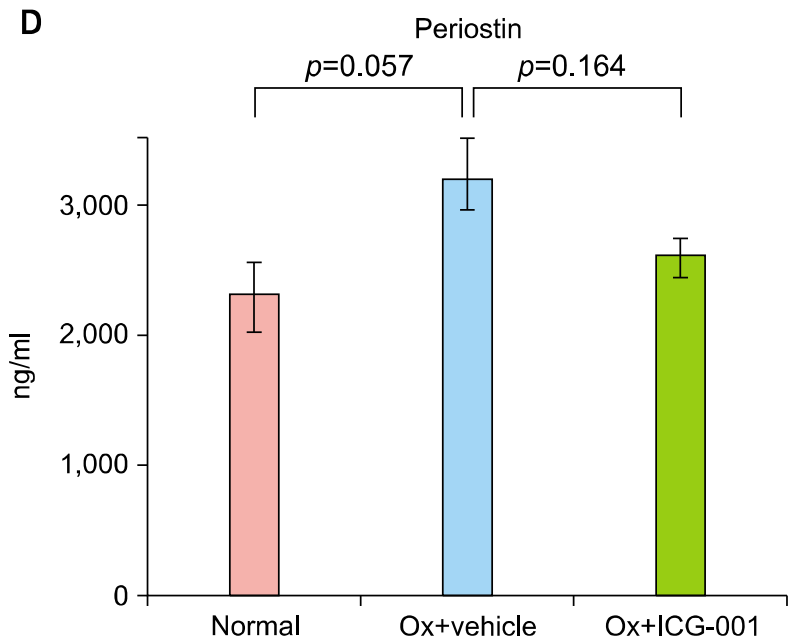

Fig. 4. Effect of ICG-001 on the serum levels of immunoglobulin E (IgE), thymic stromal lymphopoietin (TSLP), thymus and activation-regulated chemokine (TARC), and periostin. ICG-001 treatment of atopic dermatitis (AD) mice suppressed serum TSLP and TARC levels $(B, C)$, and tended to suppress serum IgE and periostin levels, without significance (A, D). Control group (normal) ( $n=4)$, Vehicle only mice (oxazolone [Ox]+vehicle) $(\mathrm{n}=8)$, ICG-001 treatment $(\mathrm{Ox}+\mathrm{ICG}-001)(\mathrm{n}=8) .{ }^{*} 0.05>p>0.01,{ }^{* *} p<0.01$.

with GATA-3 subsequently mediating the differentiation of Th2 cells $^{19}$. As previously mentioned, ICG-001 inhibits the binding of $\beta$-catenin and CBP by competitive action, increasing the binding of $\beta$-catenin to $\mathrm{p} 300$, so that ICG001 might contribute to the promotion of Th2 responses. Meanwhile, the emergence of AD-like dermatitis was significantly prevented without alteration of serum IgE levels, indicating that IgE itself may not contribute to the pathogenesis of $\mathrm{AD}$ or suggesting that ICG-001 might target pathological pathways not related to Th2 in AD-like dermatitis.

The decrease in TEWL and the increase in SC hydration by ICG-001 treatment suggest that ICG-001 might directly contribute to normalizing permeability barrier function, al- though the roles of $\beta$-catenin/CBP-dependent signaling in permeability barrier homeostasis have not been elucidated. Moreover, possible effects of ICG-001 on a variety of cells including immunologic cells such as $\mathrm{T}$ lymphocytes remains unclear.

PRI-724, an isomer or enantiomer of ICG-001, has already been used in clinical trials for a variety of malignant hematopoietic diseases, cancers, and liver cirrhosis, demonstrating the safety and tolerance of PRI-724 without significant adverse effects ${ }^{20,21}$. Taken together, $\beta$-catenin/CBP-dependent signaling might be contributed in the pathogenesis of $A D$, and could also serve as promising therapeutic targets especially by accounting for Th2 independent pathways. Further studies on human AD should be conducted for the 
therapeutic application of the blocking of $\beta$-catenin/CBPdependent signaling.

\section{ACKNOWLEDGMENT}

The authors profoundly appreciate Ms. Yoko Miyanari, Ms. Tomomi Harada, and Ms. Mika Kasagi for their skilled technical assistance.

The present study received a research grant from Sanofi K.K. Sanofi K.K. had no involvement in study design; collection, analysis and interpretation of data; and writing the manuscript.

\section{SUPPLEMENTARY MATERIALS}

Supplementary data can be found via http://anndermatol. org/src/sm/ad-31-631-s001.pdf.

\section{CONFLICTS OF INTEREST}

The authors have nothing to disclose.

\section{ORCID}

Haruna Matsuda-Hirose, https://orcid.org/0000-0003-4821-4406

Tomoko Yamate, https://orcid.org/0000-0002-8586-7783

Mizuki Goto, https://orcid.org/0000-0001-7225-5270

Akira Katoh, https://orcid.org/0000-0003-4517-7670

Hiroyuki Kouji, https://orcid.org/0000-0003-1908-8578

Yuya Yamamoto, https://orcid.org/0000-0002-9351-0856

Takashi Sakai, https://orcid.org/0000-0001-7128-3237

Naoto Uemura, https://orcid.org/0000-0002-3722-8979

Takashi Kobayashi, https://orcid.org/0000-0002-9069-5736

Yutaka Hatano, https://orcid.org/0000-0002-1349-755X

\section{REFERENCES}

1. Kabashima K. New concept of the pathogenesis of atopic dermatitis: interplay among the barrier, allergy, and pruritus as a trinity. J Dermatol Sci 2013;70:3-11.

2. Elias PM, Hatano Y, Williams ML. Basis for the barrier abnormality in atopic dermatitis: outside-inside-outside pathogenic mechanisms. J Allergy Clin Immunol 2008;121:13371343.

3. Leung DY. Atopic dermatitis: the skin as a window into the pathogenesis of chronic allergic diseases. J Allergy Clin Immunol 1995;96:302-318; quiz 319.

4. Yamaguchi Y. Periostin in skin tissue and skin-related diseases. Allergol Int 2014;63:161-170.

5. Blauvelt A, de Bruin-Weller M, Gooderham M, Cather JC, Weisman J, Pariser D, et al. Long-term management of moderate-to-severe atopic dermatitis with dupilumab and concomitant topical corticosteroids (LIBERTY AD CHRONOS): a 1-year, randomised, double-blinded, placebo-controlled, phase 3 trial. Lancet 2017;389:2287-2303.

6. Takahashi-Yanaga F, Kahn M. Targeting Wnt signaling: can we safely eradicate cancer stem cells? Clin Cancer Res 2010;16:3153-3162.

7. Clevers $H$, Nusse R. Wnt/ $\beta$-catenin signaling and disease. Cell 2012;149:1192-1205.

8. Emami $\mathrm{KH}$, Nguyen $\mathrm{C}$, Ma $\mathrm{H}$, Kim DH, Jeong KW, Eguchi $\mathrm{M}$, et al. A small molecule inhibitor of beta-catenin/CREBbinding protein transcription [corrected]. Proc Natl Acad Sci U S A 2004; 101:12682-12687.

9. Beyer C, Reichert H, Akan H, Mallano T, Schramm A, Dees C, et al. Blockade of canonical Wnt signalling ameliorates experimental dermal fibrosis. Ann Rheum Dis 2013;72:12551258.

10. Gang EJ, Hsieh YT, Pham J, Zhao Y, Nguyen C, Huantes S, et al. Small-molecule inhibition of $\mathrm{CBP} /$ catenin interactions eliminates drug-resistant clones in acute lymphoblastic leukemia. Oncogene 2014;33:2169-2178.

11. Koopmans T, Crutzen S, Menzen MH, Halayko AJ, Hackett $\mathrm{TL}$, Knight DA, et al. Selective targeting of CREB-binding protein/B-catenin inhibits growth of and extracellular matrix remodelling by airway smooth muscle. Br J Pharmacol 2016; 173:3327-3341.

12. Hatano $Y$, Man MQ, Uchida $Y$, Crumrine D, Mauro TM, Feingold KR, et al. Murine atopic dermatitis responds to peroxisome proliferator-activated receptors alpha and beta/ delta (but not gamma) and liver $\mathrm{X}$ receptor activators. J Allergy Clin Immunol 2010;125:160-169.e1-5.

13. Man MQ, Hatano $Y$, Lee $S H$, Man M, Chang S, Feingold $\mathrm{KR}$, et al. Characterization of a hapten-induced, murine model with multiple features of atopic dermatitis: structural, immunologic, and biochemical changes following single versus multiple oxazolone challenges. J Invest Dermatol 2008; 128:79-86.

14. Gaddis M, Gerrard D, Frietze S, Farnham PJ. Altering cancer transcriptomes using epigenomic inhibitors. Epigenetics Chromatin 2015;8:9.

15. Hatano Y, Adachi Y, Elias PM, Crumrine D, Sakai T, Kurahashi $R$, et al. The Th2 cytokine, interleukin-4, abrogates the cohesion of normal stratum corneum in mice: implications for pathogenesis of atopic dermatitis. Exp Dermatol 2013;22:30-35.

16. Zhang W, Sakai T, Hatano Y, Fujiwara S. Betamethasone butyrate propionate inhibits the induction of thymic stromal lymphopoietin in cultured normal human keratinocytes. Ann Dermatol 2016;28:772-775.

17. Akcora BÖ, Storm G, Bansal R. Inhibition of canonical WNT signaling pathway by $\beta$-catenin/CBP inhibitor ICG-001 ameliorates liver fibrosis in vivo through suppression of stromal CXCL12. Biochim Biophys Acta Mol Basis Dis 2018; 1864:804-818.

18. Kinoshita $H$, Takai T, Le TA, Kamijo S, Wang XL, Ushio $H$, et al. Cytokine milieu modulates release of thymic stromal lymphopoietin from human keratinocytes stimulated with 
double-stranded RNA. J Allergy Clin Immunol 2009;123: 179-186.

19. Gattinoni L, Ji Y, Restifo NP. Wnt/beta-catenin signaling in T-cell immunity and cancer immunotherapy. Clin Cancer Res 2010;16:4695-4701.

20. Krishnamurthy N, Kurzrock R. Targeting the Wnt/beta-catenin pathway in cancer: update on effectors and inhibitors. Cancer Treat Rev 2018;62:50-60.

21. Nishikawa K, Osawa Y, Kimura K. Wnt/B-Catenin Signaling as a potential target for the treatment of liver cirrhosis using antifibrotic drugs. Int J Mol Sci 2018;19:E3103. 